\title{
A Fuzzy Neural Approach with Multiple Models to Time- Dependent Short Term Power Load Forecasting Based on Weather
}

\author{
Donghui Shi ${ }^{1}$, Jian Guan ${ }^{2}$, Jozef Zurada ${ }^{2}$, Jorge Lopez-Vargas ${ }^{3}$ and Ma. Carmen \\ Cabrera-Loayza ${ }^{3}$ \\ ${ }^{1}$ Department of Computer Engineering, School of Electronics and Information \\ Engineering, Anhui Jianzhu University, Hefei, China 230601 \\ Prometeo researcher, Universidad Técnica Particular de Loja, Loja, Ecuador \\ ${ }^{2}$ Department of Computer Information Systems, College of Business, University of \\ Louisville, Louisville, KY 40292, United States \\ ${ }^{3}$ Departamento de Ciencias de la Computación, Universidad Técnica Particular \\ de Loja, San Cayetano Alto S/N, 1101608, Loja, Ecuador \\ ${ }^{1}$ sdonghui@gmail.com, ${ }^{2}$ jeff.guan@louisville.edu, ${ }^{2}$ jozef.zurada@louisville.edu \\ jalopez2@utpl.edu.ec, ${ }^{3}$ mccabrerax@utpl.edu.ec
}

\begin{abstract}
With the advancement of the big data technology, making use of the relevant data from different sources to predict the power load can become a more feasible task for power system administration. This paper presents a new adaptive neuro-fuzzy inference system (ANFIS) approach with multiple models to predict time-dependent power load values based on the weather data collected from different regions. The results show that averaging the predicted values of 10 repeated runs generates the best prediction results using average weather data as input variables. Thus the load curves with more repeated runs have better general performances. In addition the load curves on holidays show better predictability than those on work-days. The results also show that more input records may not improve the prediction accuracy. Finally the control surface generated by ANFIS reveals the impact of temperature and vapour pressure on the power load. The paper concludes that the new neuro-fuzzy approach with multiple models is a viable method for power load forecasting based on weather data.
\end{abstract}

Keywords: power load forecasting, ANFIS, weather, prediction curve

\section{Introduction}

Short term power load forecasting is very important to power system administration in China. A more precise prediction can help administrators make better power running plans and thus avoid the over-generation or under-generation of energy by power plants. In recent years, computational intelligent methods have been introduced in power load forecasting, and it has been demonstrated that the use of such methods can improve the prediction accuracy of load forecasting.

Kown et al. [1] used the Backpropagation learning algorithm for the hourly load demand forecast of a day ahead. In the paper, weather factors with a weight coefficient are taken as input variables. The results show that the error percentage is clearly reduced. Khwaja et al. [2] applied bagged neural networks for predicting the electricity load based on the dataset in which variables such as the dry bulb temperature, dew point temperature, hour of the day, and day of the week are used as input. The bagging process reduces the estimation errors compared to using a single neural network. Bahrami et al. [3] proposed a hybrid method composed of the wavelet transform and grey model improved by particle 
swarm optimization algorithm. The mean temperature, the mean relative humidity, the mean value of the wind speed, and the load data of the previous days are used as the input variables. The experiment results confirm that the method has an improved performance compared with other methods. Xiao et al. [4] proposed a new combined model to absorb the merits and avoid the limitations of the individual models. The paper claims that the proposed combined model has better performance when compared with the individual forecasting models. He et al. [5] used the risk analysis of the land-use change and load density to construct the power load forecasting model. The case study results show that this model is viable.

A novel prediction method proposed by Zhang Hong Liang [6] based on a Markov chain model with a fuzzy probability was used for long term electric power demand. Lee, Woo-Joo and Jinkyu Hong [7] used a hybrid dynamic including air temperature dependency of power load and fuzzy time series model for the mid-term electric power load forecasting. The model shows better performance with only less than $3 \%$ absolute error when compared to other models from previous studies. Han et al. [8] used clients' socio-economic factors as input variables by applying the random forests model to forecast daily electricity usage patterns. The socio-economic factors include household profiles, residential behavioral related features, housing conditions, properties of main electrical appliances. Day et al. [9] stated that the weather data is considered the most influencing factor in daily energy usage. They use usage data and weather data for developing a recommended load prediction model. Usage data are collected from customer smart meters including kilowatt usage per various time intervals and smart appliance usage.

The adaptive neural fuzzy inference system has been successfully used in classification and prediction problems in many other areas [10,11]. Researchers have started to use ANFIS models in power load forecasting. For example, the study by Zahin et al. [12] compared ANFIS with ANN. The results indicate that ANFIS is a superior method to tackle forecasting of power generation from different error measures. Rothe et al. [13] used ANFIS to predict the load demand for the next hour based on input parameters at the current hour based on the weather. Ucenic et al. [14] compared the results of ANFIS with those of an Autoregressive model (AR) and an Autoregressive Moving Average model (ARMA). The results show that the ANFIS model performs better than AR and ARMA models. Abraham et al. [15] used a fuzzy neural network to predict the power load. The input variables for the model are temperature, previous day's demand, season, day of the week, and a value expressing the $0.5 \mathrm{~h}$ period of the day. The data were recorded every half an hour for a ten-month period. The results show that the fuzzy neural network has better results than other techniques in terms of the RMSE error and performance time. The study by Kumar and Kapíl [16] used fuzzy logic and artificial neural network for short term load forecasting, in which historical hourly load demand over a week, and weather data including temperature, humidity, wind speed are used as input variables. The study [17] by Abdoos et al. proposed a new hybrid intelligent method for short term load forecasting, in which Wavelet Transform (WT) as a powerful signal analyzer is applied for the signal decomposing of electricity load. In this method, load and temperature of previous days are used for prediction of the next hour electrical load data. Osório et al. [18] integrated existing models including wavelet transform, evolutionary particle swarm optimization, and the adaptive neuro-fuzzy inference system to obtain a significant improvement results compared with other methods.

In addition, alternative energy is one of the related fields in which ANFIS model has been used to construct the prediction model of power load. For example, wind energy is increasing its participation as a main source of energy in power grids [19]. Some researchers used ANFIS model in the wind energy. Foley et al. [19] applied ANFIS to forecast the average hourly wind speed. They compared different types and number of inputs, training and checking sizes, as well as type and number of membership functions. 
Pedro et al. [20] compared several forecasting techniques for predicting the solar power output. The results show that the ANN-based forecasting models perform better than the other forecasting techniques. Chang et al. [21] used the adaptive fuzzy inference system for short-term solar power forecasting. The first layer (fuzzy layer) has 4 inputs: the solar power output of 20 minutes ago, the solar power output of 10 minutes ago, the current solar power output, and the current solar radiation value. The output layer has one output for the 10 minutes ahead solar power forecasting. The results demonstrate the effectiveness of the proposed forecasting method.

From this brief literature review it is obvious that the ANFIS model has better performance in improving the prediction accuracy. However, there is also recognition that the need exists to develop more efficient and accurate load forecast models [13]. In all the above applications and research using the ANFIS approach, only one ANFIS model was constructed for predicting the power load using time variable as one of the input variables. Because the load value of every time point is apparently different, using only one model is very difficult to precisely predict the power load. The study [22] by Souzanchi et al. used a multiple adaptive neuro fuzzy inference system to improve the power load forecasting. There is one separate adaptive neural-fuzzy system for each season, that is, four models for different seasons are used in the simulation. Except for improving the prediction accuracy, it has another advantage that the run time of system is reduced due to a decreased size in the training and validation data. The results show that the ANFIS with multiple models is a valid method in power load forecasting. In this study we propose a novel approach, which constructs 96 ANFIS models based on subsets from 96 different time points, to predict time-dependent power load values.

Fay et al. [23] presented a preliminary analysis of Irish electrical load data and analyzed the correlation between load and temperature. The data were obtained from the Electricity Supply Board (ESB) and the Meteorological Office of Ireland. In the study, other input variables of the dataset are the day, the season, and the shape of the load curve. Studies have shown that weather factors have a direct influence on the power load. Other studies also show the similar results [24]. However, in many situations, the weather data are from different regions. In this paper we show one solution to use the weather data collected from the different regions as the input to the ANFIS model. The weather data of three districts computed from the sixteen regions are used as input in Scenario 1, and the averaged provincial weather data are used in Scenario 2. We compare their performance of the models constructed using different weather data as input.

The purpose of our research is to explore the effectiveness of a neuro-fuzzy approach with multiple models for time-dependent power load forecasting based on weather data collected from different regions and verify how weather factors, including temperature, humidity, vapour pressure, and peak gust speed impact the power load.

The paper is organized as follows. Section 2 provides the data description and Section 3 presents the basic characteristics of the ANFIS models used in the study. Section 4 discusses the experiments and simulation results of the ANFIS models. Finally, Section 5 concludes the paper and proposes some probable directions for future work.

\section{Data Description}

All the power load data and weather data in the study are obtained from Anhui province, China. The power load data variables include Date, Time, and Power load. The weather data properties include Temperature, Temperature Variation, Humidity, Humidity Variation, Vapour Pressure, Vapour Pressure Variation, Peak Gust Speed, and Peak Gust Speed Variation. Figure 1 shows the preprocessing of weather forecasting data. Table 1 shows a brief description of the data set with sample data. The power load data were captured every fifteen minutes, and the weather data were captured each hour. In order to keep their consistency, we used the linear interpolation method to impute the missing data. For example, if the temperatures of the $n^{\text {th }}$ and the $(n+i)^{\text {th }}$ time points are known and 
the temperature of the intermediate time point $(n+j)^{\text {th }}$ is missing, we can use the following formula to compute the missing values:

$$
T_{n+j}=T_{n+} \frac{T_{n+i}-T_{n}}{i} ; j ; 0<j<i
$$

The weather data set records the weather forecasting data from sixteen city-regions. We average the data to form the weather data of three different districts: Huaibei, Jiangnan and Jianghuai. The total power load values of the sixteen city-regions last year were used as the weights. We use $\mathrm{T}_{1}, \mathrm{TV}_{1}, \mathrm{H}_{1}, \mathrm{HV}_{1}, \mathrm{VP}_{1}, \mathrm{VPV}_{1}, \mathrm{PGS}_{1}$ and $\mathrm{PGSV}_{1}$ to represent Temperature, Temperature variation, Vapour pressure, Vapour pressure variation, Peak gust speed, and Peak gust speed variation, respectively, in Jiangnan district. Similarly, $\mathrm{T}_{2}$ etc. and $\mathrm{T}_{3}$ etc. represent those variables in Jianghuai and Huaibei districts. In the study, three districts' weather data with twenty four properties in Scenario 1 and the average weather data with eight properties in Scenario 2 are used as the input to the ANFIS models.

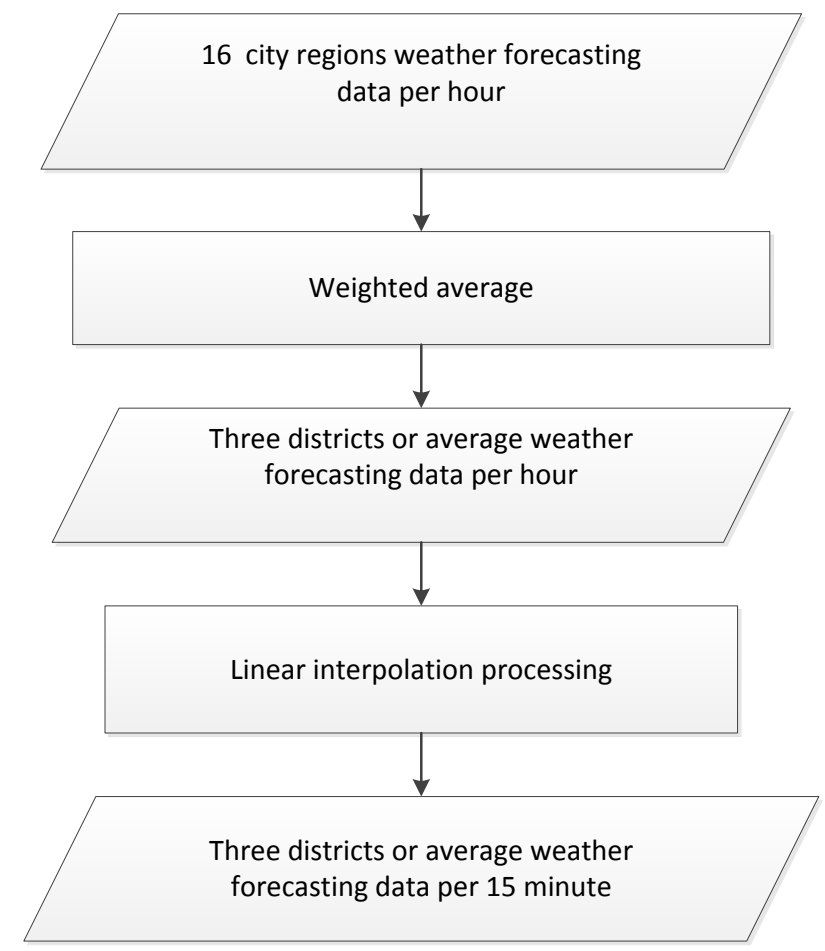

\section{Figure 1. Weather Forecasting Data Preprocessing}

Among them, the variation of the weather factors is defined as the value of the $(n+1)^{\text {th }}$ day weather factors minus the value of the $(n-1)^{\text {th }}$ day weather factors. For example, the $\mathrm{TV}_{1}$ (Temperature variation in Jiangnan District) of the (n-1) ${ }^{\text {th }}$ day, $T V_{1, n-1}$ can be calculated formulas follows:

$$
T V_{1, n-1}=T_{1, n+1}-T_{1, n-1}
$$

The power load data set and weather data sets of three districts are merged into a whole dataset for the ANFIS models to predict the future power load. The variables are described in Table 1. Power load is the output variable, the others are input variables. We transform the Time field to an integer number. For example, 1 represents 0:00 and 49 represents 12:00. 
Table 1. Variables of the Power and Weather Data Set

\begin{tabular}{|l|c|l|}
\hline Variable & Sample & Explanation \\
\hline Date & $12 / 12 / 2002$ & The current date, converted to two variables \\
\hline Time & $12: 00$ & $\begin{array}{l}\text { Current time, converted to the 49th sampling } \\
\text { point in the dataset }\end{array}$ \\
\hline Week & 6 & The current week \\
\hline Power load & 4552 & $\begin{array}{l}\text { The whole provincial power load of current } \\
\text { time in the day }\end{array}$ \\
\hline $\mathrm{T}_{1}$ & 9 & Temperature in Jiangnan \\
\hline $\mathrm{TV}_{1}$ & -5 & Temperature variation in Jiangnan District \\
\hline $\mathrm{H}_{1}$ & 71 & Humidity in Jiangnan \\
\hline $\mathrm{HV}_{1}$ & 14 & Humidity variation in Jiangnan \\
\hline $\mathrm{VP}_{1}$ & 1008 & Vapour pressure in Jiangnan \\
\hline $\mathrm{VPV}_{1}$ & -1 & Vapour pressure variation in Jiangnan \\
\hline $\mathrm{PGS}_{1}$ & 4 & Peak gust speed in Jiangnan \\
\hline $\mathrm{PGSV}_{1}$ & 1 & Peak gust speed variation in Jiangnan \\
\hline $\mathrm{T}_{2}$ & 9 & Temperature variation in Jianghuai \\
\hline $\mathrm{TV}_{2}$ & -5 & Temperature variation in Jianghuai \\
\hline $\mathrm{H}_{2}$ & 71 & Humidity in Jianghuai \\
\hline $\mathrm{HV}_{2}$ & 14 & Humidity variation in Jianghuai \\
\hline $\mathrm{VP}_{2}$ & 1008 & Vapour pressure in Jianghuai \\
\hline $\mathrm{VPV}_{2}$ & -1 & Vapour pressure variation in Jianghuai \\
\hline $\mathrm{PGS}_{2}$ & 4 & Peak gust speed in Jianghuai \\
\hline $\mathrm{PGSV}_{2}$ & 1 & Peak gust speed variation in Jianghuai \\
\hline $\mathrm{T}_{3}$ & 9 & Temperature in Huaibei \\
\hline $\mathrm{TV}_{3}$ & -3 & Temperature variation in Huaibei \\
\hline $\mathrm{H}_{3}$ & 49 & Humidity in in Huaibei \\
\hline $\mathrm{HV}_{3}$ & 10 & Humidity variation in Huaibei \\
\hline $\mathrm{VP}_{3}$ & 755 & Vapour pressure in Huaibei \\
\hline $\mathrm{VPV}_{3}$ & 2 & Vapour pressure variation in Huaibei \\
\hline $\mathrm{PGS}_{3}$ & 3 & Peak gust speed in Huaibei \\
\hline $\mathrm{PGSV}_{3}$ & & Peak gust speed variation in Huaibei \\
\hline & & \\
\hline
\end{tabular}

\section{Methodology}

Figure 2 shows the architecture of the power load forecasting model. First, we extracted the 96 small data sets along with their 96 time points from the whole dataset we have preprocessed. There are 96 time points in one day because each 15 minute has a sampling record. Second, we constructed the 96 ANFIS models based on the extracted 96 data sets. Last, we used the 96 ANFIS models to predict the power load at 96 time points. The ANFIS model was created using MatLab Fuzzy Logic Toolbox. 


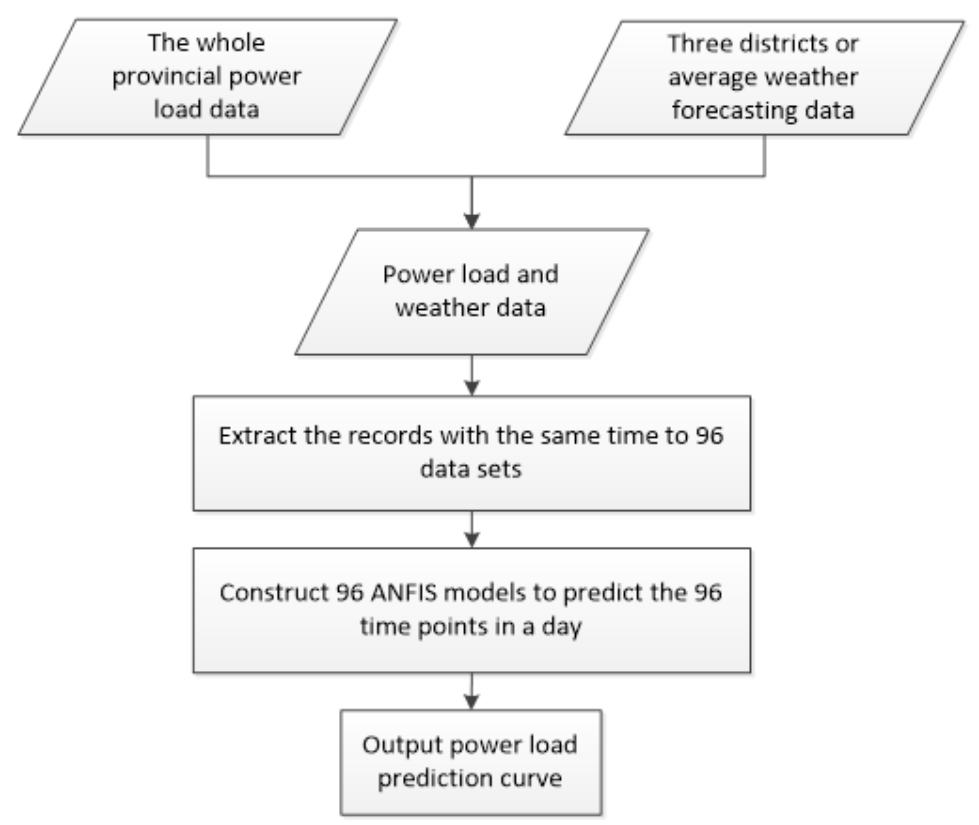

Figure 2. Architecture of the Power Load Forecasting Model

The ANFIS component computes the overall output $(\mathrm{F})$ of the model as the sum of all the weighted outputs of the previous layer. We use two scenarios to compare the performance with different input variables. In Scenario 1, there are 28 input variables as shown in Table 1. The input variable Time was not used in the ANFIS model as each model represents a time point. The model has $27(\mathrm{~m})$ input variables representing the power load and weather forecasting data described in the last section. Each of the input variables has 7 (n) membership functions. In Scenario 2, we average the weather data from three different regions to end up with 11 input variables, including Temperature, Temperature variation, Vapour pressure, Vapour pressure variation, Peak gust speed, and Peak gust speed variation. The model has $11(\mathrm{~m})$ input variables representing the power load and weather forecasting data. Each of the input variables has 7 (n) membership functions. The model has 5 layers with a Takagi, Sugeno, and Kang (TSK) type fuzzy inference system [25, 26], which is presented in Figure 3.

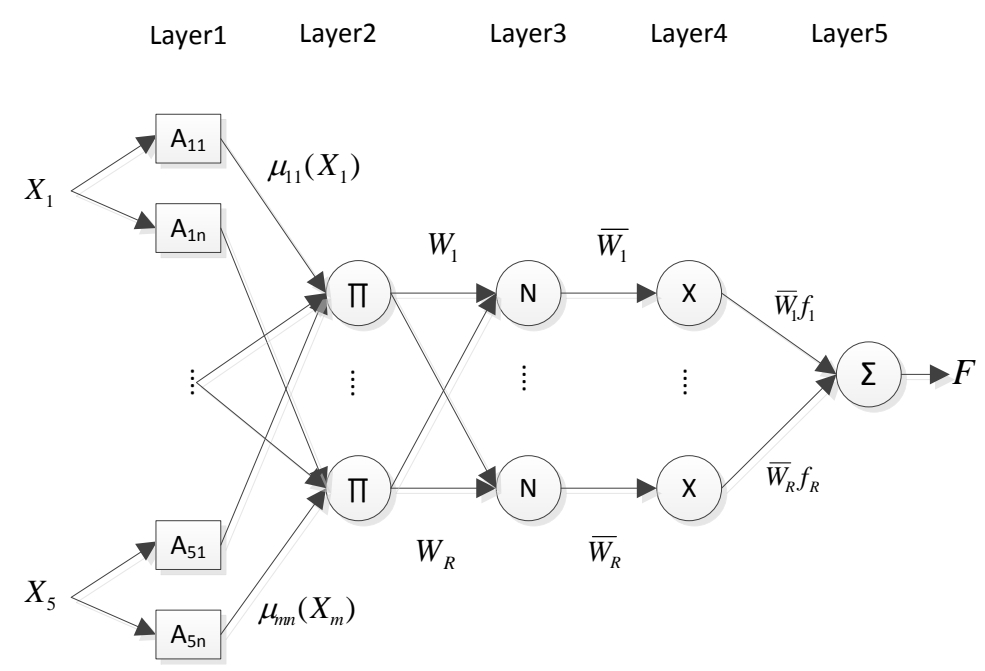

Figure 3. ANFIS Prediction Model 
Layer 1: This layer contains the membership functions with adaptive parameters or premise parameters. The number of nodes $(N)$ in the first layer is the product of the input size and the number of the membership functions for each input variable, or $N=m \times n$. The output of each node is defined as:

$$
O_{i j}=\mu_{i j}\left(X_{i}\right) \text {, for } i=1, \text { m and } j=1, n
$$

where $\mu_{i j}$ is the $j^{\text {th }}$ membership Gaussian function for the input $X_{\mathrm{i}}$ and is given as follows:

$$
\mu(X)=\exp \left\{\frac{-0.5(x-c)^{2}}{\sigma^{2}}\right\}
$$

where $\mathrm{c}$ is the mean and $\sigma$ is the variance. They are the premise parameters.

Layer 2: This layer calculates the firing strength of each rule and the output in this layer represents these firing strengths. The output is the product of all of its inputs as follows:

for $k=1, R$ and $R$ is the number of rules.

$$
O_{k}=W_{k}=\mu_{1, i}\left(X_{1}\right) \mu_{2, i}\left(X_{2}\right) \cdots \mu_{m, i}\left(X_{m}\right)
$$

Layer 3: This layer normalizes the weighing factor of each of the input nodes $\mathrm{k}$ as follows:

$$
O_{k}=\bar{W}_{k}=\frac{W_{k}}{W_{1}+W_{2}+\cdots+W_{R}}
$$

Layer 4: the output of this layer represents a weighted value of the first order fuzzy ifthen rule as follows:

$$
o_{k}=\bar{W}_{k} f_{k}
$$

where $f_{k}$ is the output of the kth fuzzy rule as follows:

$$
\begin{gathered}
\text { If }\left(X_{1} \text { is } A_{11}\right) \text { and }\left(X_{2} \text { is } A_{22}\right) \text { and } \cdots\left(X_{m} \text { is } A_{m n}\right) \\
\text { Then } f_{k}=\sum_{i=1}^{m} p_{i j} X_{i}+r_{k}
\end{gathered}
$$

where $p_{i j}$ and $r_{k}$ are called the consequent parameters and $j=1, n$ and $k=1, R$.

Layer 5: Finally this single node layer computes the overall output $(F)$ of the ANFIS model as the sum of all the weighted outputs of the previous layer as:

$$
O=F=\sum_{k=1}^{N} \bar{W}_{k} f_{k}
$$

where $f_{k}$ represents the output of the $k^{\text {th }}$ TSK-type rules as defined in layer 4 .

Both the premise parameters and consequent parameters are learned/optimized in the training process. The hybrid parameter optimization method is used for training in the study. The hybrid method uses a combination of the least-squares and back-propagation gradient descent methods to model a training data set.

\section{The Experiment Design and Results from Computer Simulation}

Computer simulation was conducted using MatLab Fuzzy Logic Toolbox. In each scenario $70 \%$ of the records in the data set were randomly allocated as training set, whereas $30 \%$ of the records were allocated to the models' validation. Three days of the records, 12/12/2002, 12/13/2002 and 12/14/2002, were used for testing, as the purpose of our research is using history data to predict the short term power load.

In the simulation, GENFIS2 [27] was used to generate an initial structure using subtractive clustering. We used Gaussian function as membership functions of all input variables. The radii in GENFIS2 is a parameter that specifies a cluster center's range of influence in each of the data dimensions. In the experiment the radii was set to 2.0. There are seven membership functions and seven rules generated in the study. The number of the premise parameters is 2 in a membership function. In Scenario 1, the number of 
consequent parameters is 28 (the number of input variables+1, is 28 ). The number of all the parameters is $27 \times 7$ (the number of membership functions) $\times 2$ (the number of premise parameters) $+(27+1) \times 7$ (the number of rules), which is equal to 574 . In Scenario 2, the number of consequent parameters is 12 (the number of input variables +1 , is 12 ). The number of all the parameters is $12 \times 7$ (the number of membership functions) $\times 2$ (the number of premise parameters) $+(12+1) \times 7$ (the number of rules), which is equal to 259 .

In Scenario 1, three different tests were conducted. In the first test, we used 1249 records from November to December (Data set 1), which has 27 input variables including the weather data of three different regions. The predictive program ran only 1 time. In the second test, the same data set was used, but the process was repeated 10 times. The prediction value is the average of each run. In the third test, we used 4128 records from October to December (Data set 2). The process was repeated 10 times. The prediction value is also the average of each run.

In Scenario 2, the data set has 11 input variables including the average weather data. Likewise, we conducted three tests. In the first test, we used 1249 records from November to December (Data set 3 ). The predictive program ran only 1 time. In the second test, the same data set was used, but the process was repeated 10 times. The prediction value is the average of each run. In the third test we used 4128 records from October to December (Data set 4). The process was repeated 10 times. The prediction value is also the average of each run.

Table 2. Error Measures ( $\mathrm{N}=$ Number of Time Points in a Day)

\begin{tabular}{|l|l|}
\hline Error Measure & Formula \\
\hline Root Mean-squared Error (RMSE) & $\sqrt{\frac{\sum_{i=1}^{n}\left(\text { actual price }_{i}-\text { predicted }_{\text {price }}\right)^{2}}{n}}$ \\
\hline Mean Absolute Error (MAE) & $\frac{\sum_{i=1}^{n} \mid \text { actual price }_{i}-\text { predicted price }_{i} \mid}{n}$ \\
\hline Average accuracy rate (AAR) & $\frac{\sum_{i=1}^{n}\left(1-\frac{\mid \text { actual price }_{i}-\text { predicted price }_{i} \mid}{\text { actual price }_{i}}\right)}{n}$ \\
\hline
\end{tabular}

Table 2 shows the error measures used in the paper. These are the Root Mean-squared Error (RMSE), Mean Absolute Error (MAE), and Average Accuracy Rate (AAR). RMSE is expressed in the same unit as actual and predicted load. The disadvantage of RMSE is that it tends to aggregate the effect of outliers. The MAE treats errors evenly according to their magnitude. In the formulas, $n$ represents the number of time points. We extracted a record every 15 minutes in a day, so the number is 96.

Tables 3 and 4 show the RMSE, MAE and AAR results of the ANFIS models used in predicting the power load of the three test days for short term load forecasting in Scenarios 1 and 2. Figure 4, 5, 6, 7 and 8 show the power prediction curves of the three days in the different scenarios and tests. From Table 3, we can see that the MAE and AAR results of the first and second tests in Scenario 1 are better than those of the third test in Scenario 1. From Table 4, similar results can be observed. It shows that more data cannot improve the prediction accuracy and the data of the nearest days are more valuable for prediction. The MAE and AAR results of the first and second tests in Scenario 2 (see Table 4) are better than those of the first and second tests in Scenario 1 (see Table 3). However, for the data set containing 4128 records from October to December, the MAE and AAR results of the third test in Scenario 2 are worse than those of the third test in 
Scenario 1. It shows that 11 input variables with the average weather data have better prediction accuracy than 27 input variables for 1249 records from November to December, which are the data of the nearest days.

From Table 4, the RMSE, MAE and AAR on Saturday are the best in Scenario 2. For example, RMSR, MAE and AAR on Saturday, 12/14/2002, are 288, 68, and 98.53\% respectively, and are better than those on Thursday and Friday. The reason could be that Thursday and Friday are a peak work day, during which there are bigger fluctuations. The RMSE, MAE and AAR with 1 run on Friday and Saturday are worse than those with 10 repeated runs. It shows that more repeated runs could make the curve smoother and may lead to better general prediction performance.

The power load prediction curve with 10 runs on Saturday with Scenario 1 in Figure 4 is worse than that with Scenario 2 in Figure 8. It shows that using the average weather data as input variables with the data of the nearest days have better prediction performance. The power load prediction curve with 1 run on Saturday in Figure 5 is worse than that with 10 repeated runs on Saturday in Figure 8. It shows that more repeated runs can make the curve smoother and may lead to better general prediction performance and verifies that the ANFIS model in Scenario 2 with the second test has the best general prediction performance.

Table 3. The RMSE, MAE and AAR Results with Scenario 1

\begin{tabular}{|l|l|l|l|l|}
\hline Date & Week & RMSE & MAE & $\begin{array}{l}\text { Average Accuracy } \\
\text { rate(\%) }\end{array}$ \\
\hline \multicolumn{7}{|c|}{ Data set 1 with running 1 times } \\
\hline $12 / 12 / 2002$ & 4 & 568 & 137 & 96.90 \\
\hline $12 / 13 / 2002$ & 5 & 218 & 160 & 96.47 \\
\hline $12 / 14 / 2002$ & 6 & 186 & 130 & 97.17 \\
\hline \multicolumn{7}{|c|}{ Data set 1 with running 10 times } \\
\hline $12 / 12 / 2002$ & 4 & 676 & 157 & 96.54 \\
\hline $12 / 13 / 2002$ & 5 & 635 & 158 & 96.47 \\
\hline $12 / 14 / 2002$ & 6 & 458 & 108 & 97.61 \\
\hline \multicolumn{7}{|c|}{ Data set 2 with running 10 times } \\
\hline $12 / 12 / 2002$ & 4 & 785 & 203 & 95.54 \\
\hline $12 / 13 / 2002$ & 5 & 913 & 233 & 94.98 \\
\hline $12 / 14 / 2002$ & 6 & 559 & 142 & 96.88 \\
\hline
\end{tabular}

Table 4. The RMSE, MAE and AAR Results with Scenario 2

\begin{tabular}{|c|c|c|c|c|}
\hline Date & Week & RMSE & MAE & $\begin{array}{c}\text { Average Accuracy } \\
\text { rate(\%) }\end{array}$ \\
\hline \multicolumn{7}{|c|}{ Data set 3 with running 1 times } \\
\hline $12 / 12 / 2002$ & 4 & 438 & 103 & 97.77 \\
\hline $12 / 13 / 2002$ & 5 & 595 & 135 & 97.15 \\
\hline $12 / 14 / 2002$ & 6 & 495 & 124 & 97.24 \\
\hline \multicolumn{7}{|c|}{ Data set 3 with running 10 times } \\
\hline $12 / 12 / 2002$ & 4 & 429 & 106 & 97.61 \\
\hline $12 / 13 / 2002$ & 5 & 358 & 92 & 98.02 \\
\hline $12 / 14 / 2002$ & 6 & 288 & 68 & 98.53 \\
\hline \multicolumn{7}{|c|}{ Data set 4 with running 10 times } \\
\hline
\end{tabular}




\begin{tabular}{|l|l|l|l|l|}
$12 / 12 / 2002$ & 4 & 1427 & 298 & 93.60 \\
\hline $12 / 13 / 2002$ & 5 & 1875 & 383 & 91.96 \\
\hline $12 / 14 / 2002$ & 6 & 1464 & 320 & 93.10 \\
\hline
\end{tabular}

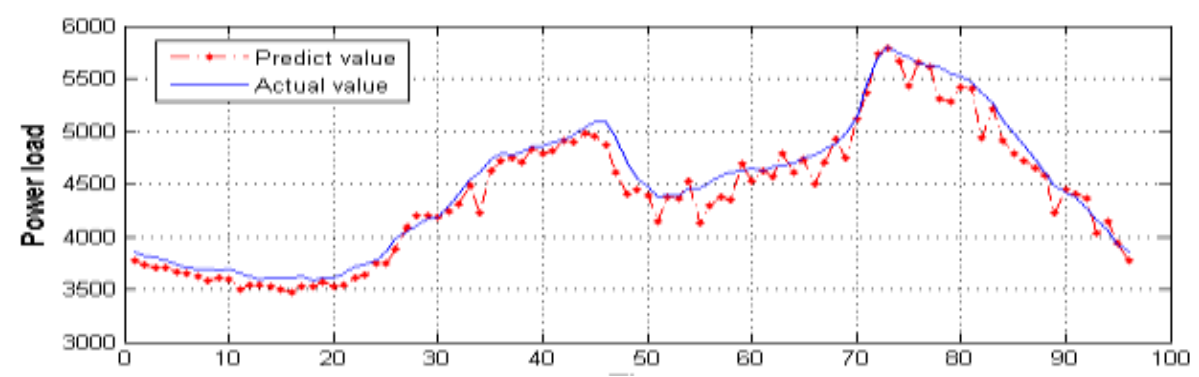

Figure 4. The Power Load Prediction Curve on 12/14/2002 in Scenario1 with the Data Set 1 and Running10 Times

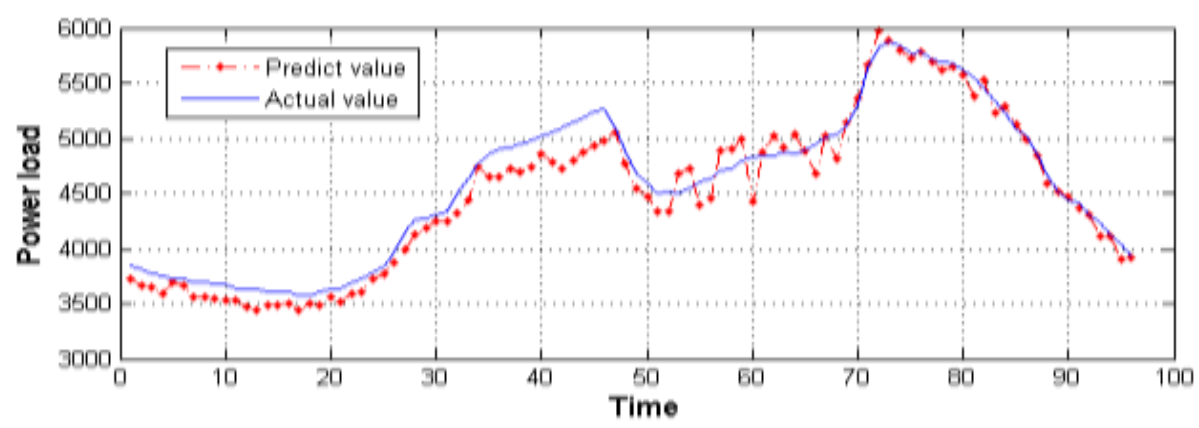

Figure 5. The Power Load Prediction Curve on 12/14/2002 in Scenario2 with the Data Set 3 and Running1 Time

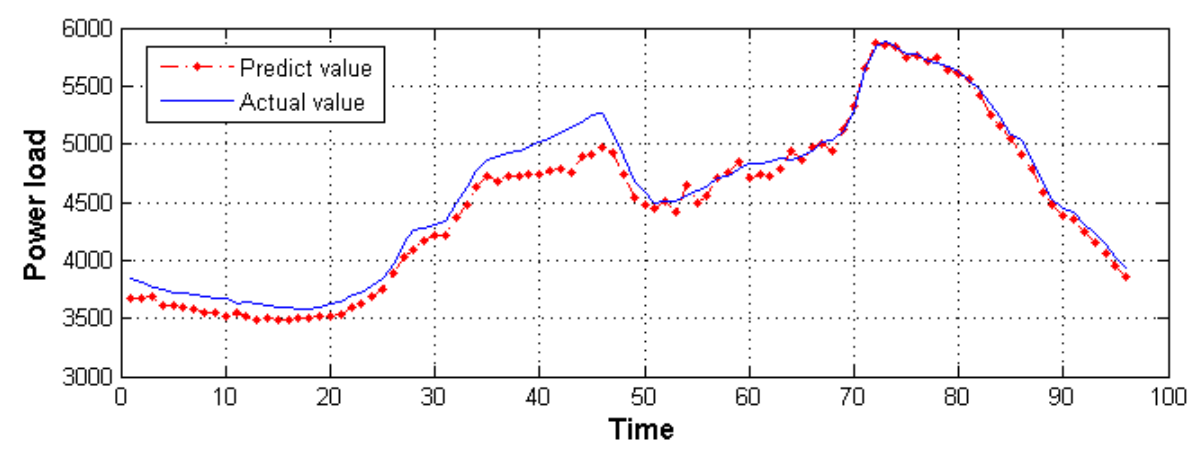

Figure 6. The Power Load Prediction Curve on 12/12/2002 in Scenario2 with the Data Set 3 and Running 10 Times 


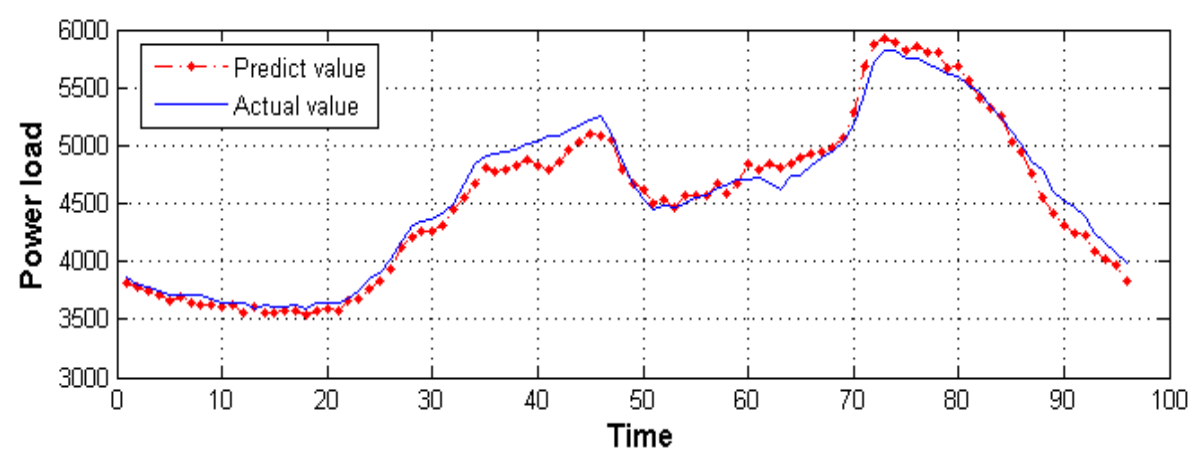

Figure 7. The Power Load Prediction Curve on 12/13/ 2002 in Scenario 2 with the Data Set 3 and Running10 Times

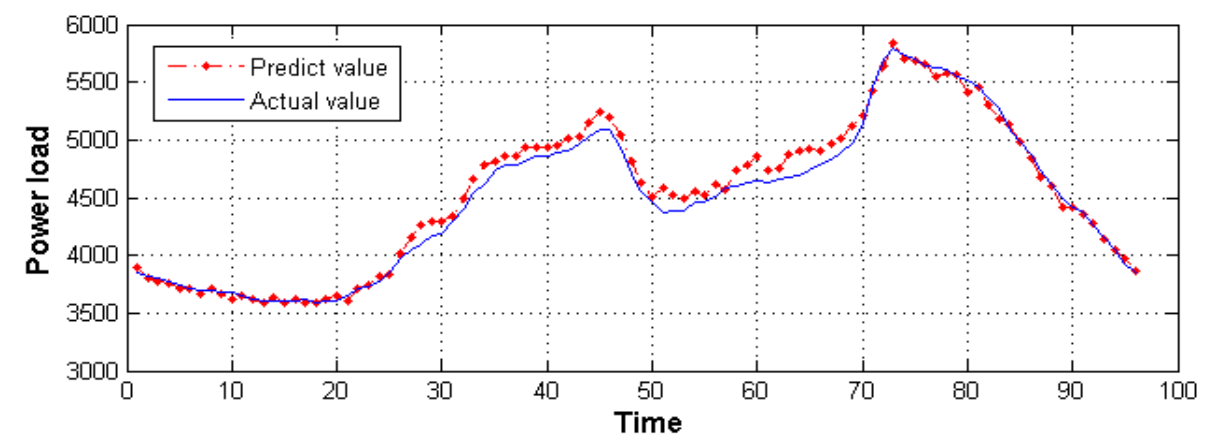

Figure 8. The Power Load Prediction Curve on 12/14/2002 in Scenario2 with the Data Set 3 and Running10 Times

The control surface depicts six 3-dimensional surfaces generated by the ANFIS model. It offers more insight into nonlinear and complex interactions between the variables and the power load. Figure 9 shows the control surface of Temperature vs. Vapour pressure at 23:45 on the date of $12 / 14 / 2002$. It shows that Temperature has a great impact on the power load. Figure10 shows the seven membership functions of Temperature at 23:45 on 12/24/2002. The membership functions for Temperature input variable in the curve are distinct. It suggests that Temperature is an important input variable. 


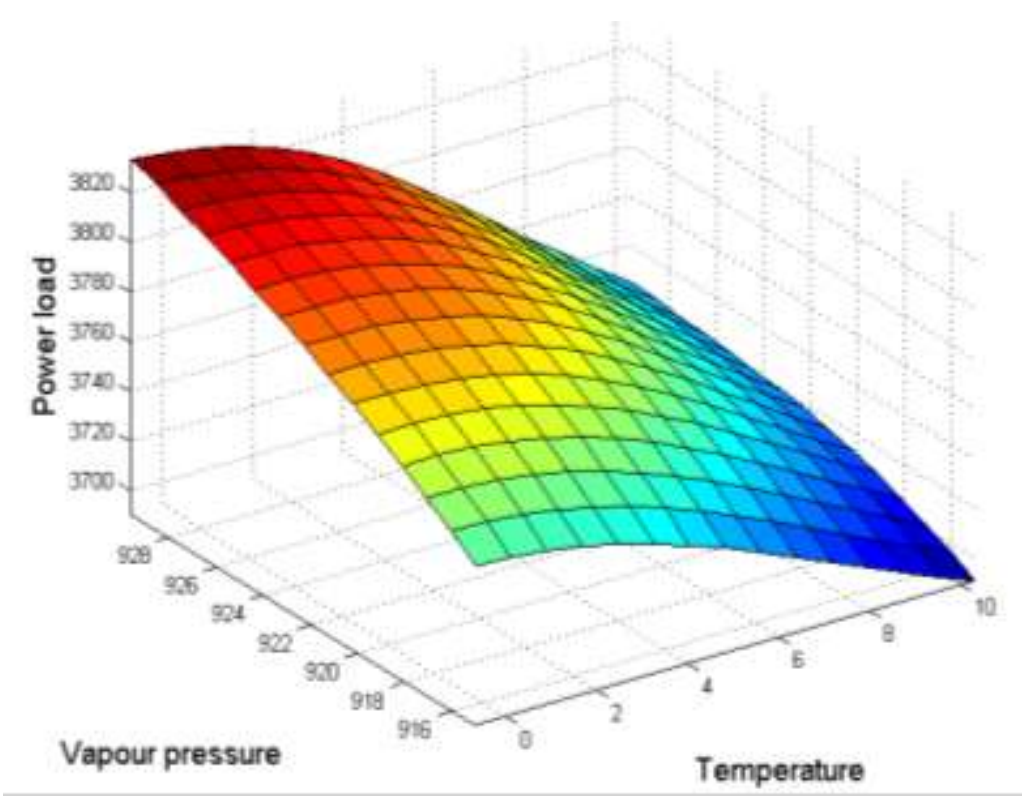

Figure 9. The Control Surface of Temperature vs. Vapour Pressure at 23:45 on 12/14/2002 with Scenario 2

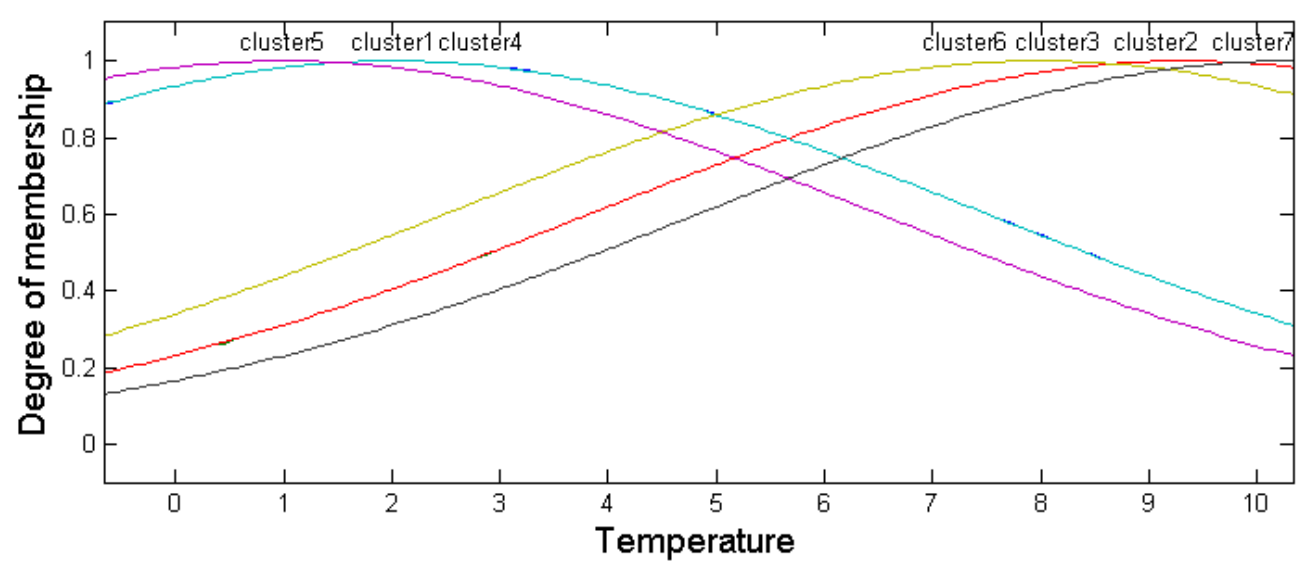

Figure 10. The Membership of Temperature at 23:45 on 12/14/2002 with Scenario 2

\section{Conclusion}

This paper presents ANFIS models for power load prediction using real power data from Anhui province, China. Computer simulation shows that our new proposed neurofuzzy approach with multiple models for power load forecasting is a viable option. The model uses a hybrid method for parameters' optimization. The computer simulation indicates that using average weather data as input variables, with the data of the nearest days and with 10 runs, generate the best prediction results. The average accuracy rates of three days are $97.61 \%, 98.02 \%$, and $98.53 \%$. Using the weather data from the three districts as input variables lead to the worse results. The reason could be that there exists dependency relationship between the weather data from the three districts. The load curves with more repeated runs have better general performances. However, more data records did not improve the prediction performance. The control surface and rules generated by ANFIS can help us understand the weather influence on the power load. Due 
to the reduced size of the training and validation data in the simulation of the ANFIS with multiple models, the run time of system is reduced greatly. Future studies may explore the relationship between weather data and power load using stream data mining.

\section{Acknowledgments}

This work was partially supported by (1) the Prometeo Project of the Secretariat for Higher Education, Science, Technology and Innovation of the Republic of Ecuador, (2) Anhui Provincial Natural Science Foundation of China (1508085MF114), and (3) Technology Foundation for Selected Overseas Chinese Scholar (2014).

\section{References}

[1] D. Kown, M. Kim, C. Hong, and S. Cho, "Short Term Load Forecasting based on BPL Neural Network with Weather Factors", International Journal of Multimedia and Ubiquitous Engineering, vol. 9, no. 1, (2014), pp. 415-424.

[2] A. S. Khwaja, M. Naeem, A. Anpalagan, A. Venetsanopoulos and B. Venkates, "Improved short-term load forecasting using bagged neural networks", Electric Power Systems Research, vol. 125, (2015), pp. 109-115.

[3] B. Saadat, R. A. Hooshmand and M. Parastegari, "Short term electric load forecasting by wavelet transform and grey model improved by PSO (particle swarm optimization) algorithm", Energy, vol. 72, (2014), pp. 434-442.

[4] X. Liye, J. Wang, R. Hou and J. Wu, "A combined model based on data pre-analysis and weight coefficients optimization for electrical load forecasting", Energy, vol. 82, (2015), pp. 524-549.

[5] Y. X. He, J. X. Zhang, Y. Xu, Y. Gao, T. Xia, and H. Y. He, "Forecasting the urban power load in China based on the risk analysis of land-use change and load density", International Journal of Electrical Power \& Energy Systems, vol. 73, (2015), pp. 71-79.

[6] Z. H. Liang, "A Novel Prediction Method for Electric Power Demand Based on Markov Chain Model with a Fuzzy Probability", In Advanced Materials Research, vol. 860, (2014), pp. 2560-2564.

[7] L. W. Joo and J. Hong, "A hybrid dynamic and fuzzy time series model for mid-term power load forecasting", International Journal of Electrical Power \& Energy Systems, vol. 64, (2015), pp. 10571062.

[8] H. Yi, X. Sha, E. G. Silva and P. Michiardi, "On the impact of socio-economic factors on power load forecasting", In Big Data (Big Data), 2014 IEEE International Conference, (2014), pp. 742-747.

[9] D. Patrick, M. Fabian, D. Noble, G. Ruwisch, R. Spencer, J. Stevenson and R. Thoppay, "Residential Power Load Forecasting", Procedia Computer Science, vol. 28, (2014), pp. 457-464.

[10] R. Ata and Y. Koçyigit, "An adaptive neuro-fuzzy inference system approach for prediction of tip speed ratio in wind turbines", Expert Systems with Applications, vol. 37, no. 7, (2010), pp. 5454-5460.

[11] F. Soltani, R Kerachian and E Shirangi, "Developing operating rules for reservoirs considering the water quality issues: Application of ANFIS-based surrogate models", Expert Systems With Applications, vol. 37, no. 9, (2010), pp. 6639-6645.

[12] Z. Sadia, H. H. Latif, S. K. Paul and A. Azeem, "A comparative analysis of power demand forecasting with artificial intelligence and traditional approach", International Journal of Business Information Systems, vol. 13, no. 3, (2013), pp. 359-380.

[13] J. P. Rothe, A. K. Wadhwani and S. Wadhwani, "Artificial Neural Network and ANFIS Based Short Term Load Forecasting in Real Time Electrical Load Environment", International Journal of Current Engineering and Technology, vol. 4, no. 3, (2014), pp. 1939-1944.

[14] U. Camelia and A. George, "A Neuro-fuzzy Approach to Forecast the Electricity Demand", Proceedings of the 2006 IASME/WSEAS International Conference on Energy \& Environmental Systems, Chalkida, Greece, (2006 ), pp. 299-304.

[15] A. Ajith and B. Nath, "A neuro-fuzzy approach for modelling electricity demand in Victoria", Applied Soft Computing, vol. 1, no. 2, (2001), pp. 127-138.

[16] K. Sunil and K. Dev Sharma, "Application of Neuro fuzzy in Power System for Short Term Load Forecasting”, International Electrical Engineering Journal (IEEJ), vol. 5, no. 9, (2014), pp. 1526-1530.

[17] A. Adel, M. Hemmati and A. A. Abdoos, "Short term load forecasting using a hybrid intelligent method", Knowledge-Based Systems, vol. 76, (2015), pp. 139-147.

[18] G. J. Osório, J. C. O. Matias and J. P. S. Catalão, "Short-term wind power forecasting using adaptive neuro-fuzzy inference system combined with evolutionary particle swarm optimization, wavelet transform and mutual information”, Renewable Energy, vol. 75, (2015), pp. 301-307.

[19] M. F. Aoife, P. G. Leahy, A. Marvuglia and E. J. McKeogh, "Current methods and advances in forecasting of wind power generation", Renewable Energy, vol. 37, no. 1, (2012), pp. 1-8.

[20] T. C. P. Hugo and F. M. C. Coimbra, "Assessment of forecasting techniques for solar power production with no exogenous inputs”, Solar Energy, vol. 86, no. 7, (2012), pp. 2017-2028. 
[21] C. W. Yeau and H. C. Miao, "Short-Term Solar Power Forecasting Using the Adaptive Network-Based Fuzzy Inference System", International Conference on Chemical, Material and Food Engineering, (2015), pp. 640-643.

[22] S. K. Zohreh, H. F. T. M. Yaghoubi, and T. M. R. Akbarzadeh, "A multi adaptive neuro fuzzy inference system for short term load forecasting by using previous day features", In Electronics and Information Engineering (ICEIE), International Conference, IEEE, vol. 2, (2010), pp. V2-54.

[23] F. Damien, J. V. Ringwood, M. Condon and M. Kelly, "Establishing a solution strategy for electrical demand forecasting in Ireland", In: Proceedings of the 22nd International Symposium on Forecasting, (2002).

[24] C. Y. Ping and Y. L. Chang, "The Factors Affecting Accuracy Rate of Power Load Forecasting in Summer", In Advanced Materials Research, vol. 1049, (2014), pp. 617-620.

[25] S. Michio and G. T. Kang, "Structure identification of fuzzy model", Fuzzy sets and systems, vol. 28, no. $1, \mathbf{( 1 9 8 8 )}$, pp.15-33.

[26] T. Tomohiro and M. Sugeno, "Fuzzy identification of systems and its applications to modeling and control", Systems, Man and Cybernetics, IEEE Transactions, (1985), pp. 116-132.

[27] "Fuzzy Logic Toolbox User's Guide for Use with MATLAB”, The MathWorks, Inc., (1998).

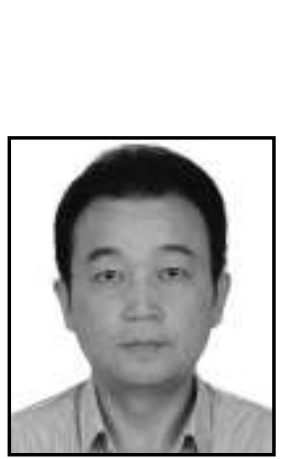

\section{Authors}

Donghui Shi, is a Professor in the Department of Computer Engineering, School of Electronics and Information Engineering, Anhui Jianzhu University, China. He received his Ph.D. in Computer Science from the University of Science and Technology of China. He was a Prometeo researcher in Universidad Técnica Particular de Loja, Ecuador and was a visiting scholar at the Department of Computer Information Systems, the College of Business, University of Louisville. His research interests are in machine learning and data mining.

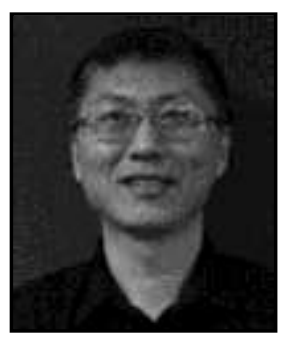

Jian Guan, is an Associate Professor in the Department of Computer Information Systems of the College of Business, University of Louisville. His research interests include accounting information systems, data mining, and knowledge management. He has published in journals such as Expert Systems with Applications, IEEE Transactions on Systems, Man, and Cybernetics, Communications of the AIS, Business Horizons, Computers in Industry, Journal of Information Systems, and Journal of Real Estate Research.

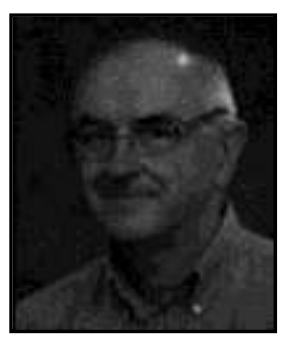

Jozef Zurada, is a Professor in the Department of Computer Information Systems, College of Business, University of Louisville, Kentucky, USA. He received his Ph.D. degree in Computer Science Engineering from University of Louisville, Kentucky, USA, in 1995 and D.Sc. (habilitation) degree from the Polish Academy of Sciences in 2014. Dr. Zurada's research interests include applications of advanced computational intelligence methods for aiding in decision making in business and manufacturing systems. He published 2 books and about 90 refereed papers and delivered over 50 presentations at international and national conferences.

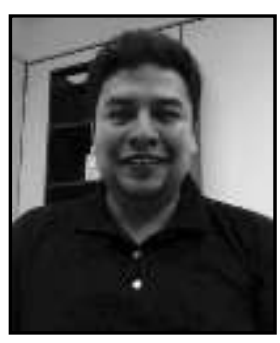

Jorge Lopez-Vargas, received the computer engineering degree from the Universidad Técnica Particular de Loja (UTPL), Loja, Ecuador, in 2004, and is currently pursuing the Ph.D. degree in software and systems with the Madrid Technical University (Universidad Politécnica de Madrid, UPM), Madrid, Spain. He is currently with the UTPL as a Professor of computer science and 
programming with the Computer Sciences Department; and he leads the Advanced Web Technologies research group and the Data Science Laboratory. His general research interests are open access, social network analysis, open educational resources and open linked data.

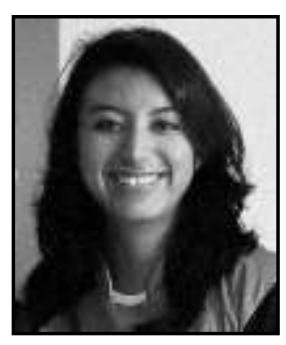

Ma. Carmen Cabrera-Loayza, is a Engineer and Computer Systems at the Universidad Técnica Particular de Loja (UTPL). Ma. Carmen Cabrera-Loayza is currently completing her studies at the Master of Artificial Intelligence at the Universidad Politécnica de Madrid. Currently she is a Professor at the School of Computer Science UTPL. Also, is a Researcher of Laboratory of Advanced Technologies of Web and Knowledge Based Systems of Compuer Science Department, UTPL. The principal research areas of CabreraLoayza are: Knowledge Representation, Semantic Web, Linked Data, and Data Science. 
International Journal of Multimedia and Ubiquitous Engineering Vol.12, No.1 (2017) 